Las técnicas del superaprendizaje como una didáctica de aprendizaje en la resolución de problemas matemáticos

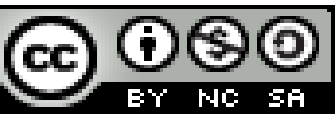

Ciencias de la educación

Artículo de investigación

\title{
Las técnicas del superaprendizaje como una didáctica de aprendizaje en la resolución de problemas matemáticos
}

\section{The techniques of super-learning as a teaching teaching in the resolution of mathematical problems}

\section{As técnicas de super-aprendizagem como ensino de ensino na resolução de problemas matemáticos}

\author{
Alba D. Alay Giler ${ }^{\mathrm{I}}$ \\ aalay@utm.edu.ec \\ Luis C. Zambrano-Villavicencio II \\ lczambrano@utm.edu.ec \\ Mirian E. Alcívar-Cruzatty II \\ mealcivar@utm.edu.ec
}

Recibido: 10 de julio de 2018 * Corregido: 05 de agosto de 2018 * Aceptado: 17 de septiembre de 2018

I. Magíster en Gerencia de Proyectos Educativos y Sociales, Licenciada en Ciencias de la Educación Espec. Física y Matemáticas, Profesor de Segunda Enseñanza Especialidad Física y Matemáticas, Docente de la Universidad Técnica de Manabí, Portoviejo, Ecuador.

II. Magíster En Gerencia Educativa, Licenciado En Ciencias de la Educación Especialidad Física y Matemáticas, Profesor de Segunda Enseñanza, Docente de la Universidad Técnica de Manabí, Portoviejo, Ecuador.

III. Magíster en Gerencia de Proyectos Educativos y Sociales, Licenciada en Ciencias de la Educación Especialización Física y Matemáticas, Docente de la Universidad Técnica de Manabí, Portoviejo, Ecuador. 


\section{Resumen}

Las técnicas de superaprendizaje permiten en el estudiante estimular el aprendizaje creando condiciones que dinamicen el aprovechamiento total de sus potencialidades. Ante esta posición, el presente artículo evaluó el manejo Constructivo de la técnica de superaprendizaje como una didáctica de aprendizaje en la resolución de problemas matemáticos. Para ello, se utilizó una metodología de tipo descriptivo y un diseño no experimental. La población la constituyeron 120 estudiantes que cursan materias del área de matemática de la Universidad Técnica de Manabí. La recolección de la información fue a través de un instrumento tipo cuestionario, con una escala tipo Licker con 3 alternativas de repuestas. Los resultados del análisis permitieron concluir que el manejo constructivo de la técnica de enseñanza de superaprendizaje, aplicada por los facilitadores, permitieron elevar la disposición del participante para contextualizar las teorías matemáticas con las cuales interactúa. Mientras que la construcción colectiva solo algunas veces se logra de acuerdo a la opinión de los facilitadores.

Palabras clave: superaprendizaje; resolución de problemas; didáctica de aprendizaje.

\section{Abstract}

The techniques of superaprendizaje allow the student to stimulate learning by creating conditions that stimulate the full use of their potential. In view of this position, the present article evaluated the construction management of the technique of superaprendizaje as a didactic of learning in the solution of mathematical problems. For this, a descriptive methodology and a non-experimental design were used. The population was made up of 120 students who study Mathematics at the Technical University of Manabí. The collection of the information was through a questionnaire-type instrument, with a Licker-type scale with 3 alternative answers. The results of the analysis allowed us to conclude that the constructive management of the teaching technique of superaprendizaje, applied by the facilitators, allowed to elevate the disposition of the participant to contextualize the mathematical theories with which it interacts. While collective construction only sometimes is achieved according to the opinion of the facilitators.

Key words: superaprendizaje; Problem resolution; learning didactics. 


\section{Resumo}

As técnicas de superaprendizaje permitem ao aluno estimular o aprendizado criando condições que estimulem o pleno aproveitamento de seu potencial. Tendo em vista esta posição, o presente artigo avaliou o gerenciamento da construção da técnica de superaprendização como uma didática de aprendizagem na solução de problemas matemáticos. Para isso, utilizou-se metodologia descritiva e delineamento não experimental. A população foi composta por 120 alunos que estudam matemática na Universidade Técnica de Manabí. A coleta das informações foi por meio de um instrumento do tipo questionário, com uma escala tipo Licker com 3 respostas alternativas. Os resultados da análise permitiram concluir que a gestão construtiva da técnica de ensino de superaprendizaje, aplicada pelos facilitadores, permitiu elevar a disposição do participante em contextualizar as teorias matemáticas com as quais interage. Enquanto a construção coletiva só às vezes é alcançada de acordo com a opinião dos facilitadores.

Palavras chave: superaprendizaje; resolução de problemas; didática de aprendizagem.

\section{Introducción}

Actualmente existen muchas técnicas que pueden ser aplicadas, para lograr aprendizajes significativos en los estudiantes, al interactuar con experiencias de resolución de problemas, estas técnicas se asocian con factores que modifican el aprendizaje en el individuo. Al respecto, Reyes (1996), explica que es una técnica que eleva las habilidades cognoscitivas de los estudiantes para la resolución de problemas, propicia una inteligencia superior, armonizando cuerpo y mente y se apoya en la capacidad de asociar ciertos elementos ambientales, físicos, intelectuales y afectivos, que al organizarse con una intención de aprendizaje eleva su nivel de logro.

De allí, que todo ser humano puede llegar a utilizar las técnicas del superaprendizaje para beneficio propio y del colectivo, el cual va pasando por diferentes etapas dependiendo de las experiencias, actualización constructiva y disposición que tenga el estudiante. Al respecto la Organización Educativa Científica y Cultural de la Naciones Unidas, UNESCO (2005):

Los países de América Latina han venido realizando una serie de acciones que permiten dar respuesta a las necesidades sociales, culturales, éticas e intelectuales del momento y propone desarrollar el 
factor intelectual como redefinición cognoscitiva que permita superar paradigmas tradicionales de dependencia instrumental y teórica (p.6).

Esta situación, exige fortalecer las configuraciones cognitivas de los estudiantes en procura de una redefinición que favorezcan su adecuación a los cambios sociales, culturales, educativos y tecnológicos a los cuales debe dar respuesta como estudiante y profesional. En efecto, se infiere la necesidad de una enseñanza diferenciable que aprovecha la estructura lógica y asociativa de los estudiantes, sin embargo, en algunos programas de formación docente, en la práctica se desvinculan los contenidos, los procesos y el contexto que limitan la capacidad de asociación entre los contenidos, ideas y experiencias.

Todo lo expuesto, adquiere importancia si se considera que la educación superior requiere: la interacción del estudiante en un contexto socializado, donde a partir de experiencias particulares y de interés, puedan ir construyendo su aprendizaje, esto amerita la participación del estudiante en la búsqueda de significado en los contenidos que intercambia y en las ideas que comparte con el facilitador. Con base en lo expuesto, en este artículo se evaluó el manejo Constructivo de la técnica de superaprendizaje como una didáctica de aprendizaje en la resolución de problemas matemáticos.

\section{Desarrollo}

Pisano (2000), explica que las complejas investigaciones llevadas a cabo en la última década sobre el mecanismo del pensamiento han originado la conocida teoría aplicada al cerebro "hemisferio izquierdo/hemisferio derecho" la cual plantea el desarrollo del pensamiento analítico, racional y lógico en el hemisferio izquierdo y la intuición, la capacidad creadora y la imaginación en el hemisferio derecho. A tal efecto, está considerado el aprendizaje como un conjunto de conocimientos a través de los cuales se establecen relaciones estímulo-respuestas. De allí que, los cambios producidos, por tanto, pueden ser deliberados o involuntarios, para mejorar o empeorar; no obstante, se convierten en aprendizaje cuando son producto de la experiencia y de la interacción de una persona con su entorno. De allí que, un entorno en el cual el participante se sienta cómodo y tomado en cuenta como persona es un ambiente propicio para el aprendizaje. 
Por su parte Pulpillo, (2001), propone como estrategia o táctica de facilitación por parte del docente “...crear el ambiente o clima para las experiencias del grupo"; mientras que Cárdenas (2005) indica la llamada teoría ecléctica o teoría del diseño instruccional, cuya preocupación fundamental es “...la creación de ambientes de aprendizaje más efectivos para los participantes”. Este hecho conduce a entender el conocimiento como “....algo complejo, globalizante, holístico, en el cual intervienen todos los procesos mentales originados en ambos hemisferios cerebrales", para ello indica Martínez (2004), se debe hacer buen manejo constructivo de las técnicas de superaprendizaje, a partir de la interacción significativa, transferencia y construcción colectiva

\section{Manejo Constructivo de las Técnicas de Superaprendizaje}

Arellano (2001), señala que el superaprendizaje o aprendizaje acelerado está orientado a mejorar las relaciones entre los participantes y facilitadores del entorno educativo, dándole significado a las experiencias personales; hace que sean conscientes de sus limitaciones y potencialidades, lo cual aunado a una serie de técnicas de control del cuerpo y mente, permite alcanzar estados de relajación y tranquilidad, ayudando así a mejorar la calidad del proceso educativo. De igual modo, se enriquece con la música, el color, la relajación, el clima apropiado en el aula y la comunicación consiente asertiva como un espacio multidimensional, con formas y características asociadas con los intereses y necesidades de los participantes, el cual puede ser representado mediante mapas mentales. Todas estas estrategias de superaprendizaje conducen a un aprendizaje holístico fundamentado en la teoría humanística, como marco principal, seguida de la neurociencia o estudio de la mente y el cerebro, lo cual abarca el cerebro triuno, los modelos de las esferas mentales, y de las inteligencias múltiples, así como el del aprendizaje transpersonal.

Al respecto Martínez (2004) afirma que la mente humana es de tal magnitud, que el hombre normal y corriente solo desarrolla entre el $5 \%$ y el $7 \%$ de sus posibilidades"(p.82). Sin embargo, este porcentaje puede hasta quintuplicar en condiciones óptimas de orquestación didáctica, es decir, aplicando técnicas para optimizar la intervención de los tres cerebros en un equilibrio armónico y de acuerdo a la materia, disciplina, asignatura o programa a enseñar.

Considera el autor que las condiciones óptimas de orquestación didáctica se lograrán en mayor o menor nivel en la medida en que se sigan más o menos si se crean un clima emocional óptima. Este permite que el docente logra en su clase un ambiente de alegría y felicidad, con ausencia de tensión, 
de estrés, de amenaza y de ansiedad. En consecuencia, la serenidad y la paz emocional, en cambio, crea las condiciones para una tranquila actividad mental, intelectual y creativa, libre de la tensión que agota y consume un alto nivel de energía.

Esto es importante atenderlo si se considera que la tensión proviene de la falta de confianza en sí mismos, en la propia capacidad para entender, memorizar y utilizar el contenido de una explicación. En efecto, parece que el mayor obstáculo que se opone a la expansión y uso de la mente reflexiva, lo que más inhibe su capacidad y dinamismo, es la falta de fe en lo que se realiza, ya que la confianza en sí mismo y en la propia capacidad para eliminar constreñimientos mentales que imposibilitan, a nivel neurofisiológico cerebral de los engramas, el flujo de ideas y sus relaciones.

El manejo constructivo y una alta disposición son necesarias para desarrollar una capacidad inmensamente mayor de la que utiliza ordinariamente. Ahora bien, esta puesta en acción depende del clima emocional que se logra establecer.

\section{Interacción Significativa}

La interacción significativa se apoya en el aprendizaje dentro de un enfoque constructivista. Pulpillo (2001), señala que el constructivismo es una teoría que equipara el aprendizaje con la creación de significados a partir de experiencias.

Los constructivistas comparten la creencia que el conocimiento no es independiente de la mente y puede ser "representado" dentro del estudiante. Estos no niegan la existencia del mundo real, pero sostienen que lo que conocemos de él, nace de la interpretación de nuestras experiencias.

En consecuencia, las representaciones internas están constantemente abiertas al cambio, no existe una realidad objetiva que los estudiantes se esfuercen en conocer. El conocimiento emerge en contextos que le son significativos, por lo tanto, para comprender el aprendizaje que ha tenido lugar en un individuo debe examinarse en su totalidad.

\section{Transferencia de Experiencias}

Es fundamental que el aprendizaje tenga lugar en ambiente reales y que las actividades de aprendizajes seleccionadas estén vinculadas con las experiencias vividas con los estudiantes.

Martínez (2004) afirma que los constructivistas creen que la mente filtra el mundo para producir su propia y única realidad. 
En efecto, los estudiantes no transfieren el conocimiento del mundo externo hacia su memoria, más bien construyen interpretaciones personales basadas en las experiencias e interacciones individuales. En este orden, Brown (2009) señala que la meta de la instrucción no asegurar que el individuo conozca hechos particulares sino más bien que pueda elaborar e interpretar información. La comprensión se desarrolla a través de la utilización continua y situacional, no se cristaliza en una definición categórica que pueda evocarse desde la memoria (p.11).

El interés del constructivismo se sitúa en la creación de herramientas cognitivas que reflejen la sabiduría de la cultura en la cual se utiliza, así como los deseos y experiencias de los individuos. Es innecesaria la mera adquisición de conceptos o detalles fijos, abstractos o auto contenidos. Esto ubica el aprendizaje en interacción con una serie de factores que explican integralmente el proceso de aprender y los elementos que lo modifican.

\section{La Construcción Colectiva}

La construcción del conocimiento requiere la interacción colectiva, implica la coordinación de actividades en las organizaciones educativas, para que de esta manera se realicen intercambios de conocimientos, experiencias y aportes que permitan el mejoramiento permanente y progresivo de cada sistema educativo.

Al respecto, Cárdenas (2005) señala que:

Mediante la aplicación de este principio de la planificación se abren espacios a todos los sectores involucrados en el quehacer educativo (docentes, estudiantes, universidades, gremios docentes, sociedad civil y medios de comunicación social) a fin de permitir su participación en la formulación del diseño curricular a través de estrategias de consulta dirigidas a estos actores, lo que genera niveles de compromiso, colaboración, receptividad y un intercambio de experiencias que enriquecen el diseño y ofrecen respuestas a las exigencias del siglo XXI.

De esta manera, se plantea la construcción colectiva de todos los sectores involucrados en el hecho educativos, para trabajar hacia un mismo fin, elevar la calidad del proceso de aprendizaje, tomando en cuenta los ejes principales que orienta la Educación Superior. Esta situación se basa en la necesidad de involucrar a todo el personal educativo para abarcar todos los elementos que interactúan en el hacer educativo, de una manera integral formar a los individuos como entes críticos, reflexivos frente a la 
realidad social. En tal sentido, es importante aplicar el consenso para la aplicación de las acciones y toma de decisiones para así unificar las ideas a implementar que cubran las necesidades de las instituciones, basándose en la participación de toda la comunidad universitarios. Los estudiantes se vinculen en un proceso de construcción del aprendizaje, a través de una serie de procedimientos que van desde el reconocimiento hasta la deducción, apoyado en la disposición de los estudiantes.

Esto es importante atenderlo, por lo planteado por Basabe, C (2006), algunos facilitadores del área de matemática aún señalan que su materia es difícil, que muy pocos alumnos le entenderán, que la mayoría fracasarán, esto crean un clima exactamente contrario a las exigencias de aprendizaje. Igualmente existen quienes colocan a los estudiantes problemas insolubles, y otros que prepara el examen con problemas que el mismo no ha resuelto, esto frustra y desestimula a los alumnos. Estos docentes no contribuyen al aprendizaje, sino se convierten en inhibidores del mismo.

Las técnicas permiten habilitar a los estudiantes que descubran nuevos conocimientos y el mejor modo de lograrlo es descubriendo por su propia cuenta algunos conocimientos ya existentes, a través de su propia acción.

Estas técnicas deben ser seleccionadas, para que sean estimulantes y coherente con las expectativas y conocimientos estén a su alcance y dentro de lo posible y para que sean previos a los estudiantes. Entre estas técnicas se encuentra: el interrogatorio, la argumentación, la descripción y le debate, estas propicia la participación del estudiante y permite validar ante el grupo las ideas y conocimientos de los estudiantes y puede ser de gran valor para el pensamiento independiente exigen un máximo de participación para la elaboración de conceptos son procedimientos didácticos fundamentalmente activo, ya que éste consiste en la discusión de un tema por parte del estudiante, bajo la dirección del docente.

\section{Metodología}

La investigación se ubicó en un estudio descriptivo y no experimental, de acuerdo a Hernández, Fernández y Baptista (2014), afirman que la investigación descriptiva es la que se orienta a redactar informes relacionados con el estado real de las personas, objeto situaciones o fenómenos tal cual se presentan en el momento de su recolección, describe lo que mide sin analizar inferencias ni verificar hipótesis. La población la constituyeron 120 estudiantes que cursan materias del área de matemática 
de la Universidad Técnica de Manabí. La recolección de la información fue a través de un instrumento tipo cuestionario, con una escala tipo lickerd con 3 alternativas de repuestas. La validez del instrumento fue realizada a través del criterio de expertos en el área y la confiabilidad se determinó a través del Coeficiente de Alfa Cronbach, cuyo resultado fue de 0.93, considerado altamente significativo. La técnica de análisis fue la estadística descriptiva y frecuencial.

\section{Resultados}

El análisis de los resultados posterior a la aplicación del instrumento que permitió obtener la información relacionada a las técnicas del superaprendizaje como una didáctica de aprendizaje en la resolución de problemas matemáticos, en la que se pudo constatar:

Tabla Nº1. Distribución Frecuencial y Porcentual del Indicador Interacción Significativa

\begin{tabular}{ccccc} 
& \multicolumn{5}{c}{ ESTUDIANTES } \\
\hline ANALISIS/ ALTERNATIVAS & $\boldsymbol{F R}$ & $\boldsymbol{\%}$ & $\boldsymbol{F R A}$ & $\boldsymbol{\%} \boldsymbol{A}$ \\
\hline SIEMPRE & 15 & 12 & 15 & 12 \\
\hline ALGUNAS VECES & 63 & 52 & 78 & 64 \\
\hline NUNCA & 42 & 36 & 120 & 100 \\
\hline 7 TOTAL & 120 & 100 & 120 & 100 \\
\hline
\end{tabular}

Fuente: Elaboración propia (2018).

En la tabla 1, se presenta la distribución frecuencial y porcentual del indicador interacción significativa, en la que se evidencio que el $52 \%$ de los investigados coincidieron en opinar que solo algunas veces se utiliza durante la interacción de clases acciones sistemáticas que permitan la construcción del aprendizaje de los alumnos, así como el establecer un orden lógico cuando se formula un problema para que puedan deslindar los componentes principales en su formulación, el aprovechar el pensamiento lógico de los estudiantes para explicar los diferentes componentes que estructuran el problema por resolver y procuran crear condiciones para que el estudiante construya su aprendizaje. Es importante destacar que la interacción significativa parte de los requerimientos de los estudiantes 
bajo la creencia que el conocimiento no es independiente de la mente y puede ser "representado" dentro del estudiante.

Tabla N²2. Distribución Frecuencial y Porcentual del Indicador Transferencia de Experiencias

\begin{tabular}{ccccc} 
& \multicolumn{5}{c}{ DOCENTES } \\
\hline ANALISIS/ ALTERNATIVAS & $\boldsymbol{F R}$ & $\%$ & $\boldsymbol{F R A}$ & $\boldsymbol{\% A}$ \\
\hline SIEMPRE & 28 & 23 & 28 & 23 \\
\hline ALGUNAS VECES & 61 & 51 & 89 & 74 \\
\hline NUNCA & 31 & 26 & 120 & 100 \\
\hline TOTAL & 120 & 100 & 120 & 100 \\
& & & & \\
\hline
\end{tabular}

Fuente: Elaboración propia (2018).

En la tabla 2, se presenta la distribución frecuencial y porcentual del indicador transferencia de experiencia en lo que respecta a si los docentes vinculan la naturaleza del problema con experiencia directa de aprendizaje y si construye sus propios programas de acción dependiendo de los conocimientos previos que tengan los estudiantes. Se evidenció, que la alternativa algunas veces se ubicó con el mayor porcentaje con un 51\%, seguida de la alternativa nunca con un $31 \%$ mientras que el menor porcentaje fue para la alternativa siempre con un $23 \%$ de las opiniones. Cabe destacar, que las experiencias de aprendizaje de los estudiantes se apoyan en la transferencia de conocimientos que permitan contextualizar los contenidos a partir de requerimientos de los estudiantes o las proposiciones de los docentes. La posición constructivista asume que las transferencias de experiencias pueden facilitarse envolviendo las personas en tareas auténticas ancladas en contextos significativos". Es decir, el conocimiento se procesa y traslada a otros contextos vinculado de forma inexorable con el conocimiento inmerso en él y con las experiencias de los estudiantes. 
Tabla 3. Distribución Frecuencial y Porcentual del Indicador Construcción Colectiva

\begin{tabular}{ccccc} 
& \multicolumn{5}{c}{ DOCENTES } \\
\hline ANALISIS/ $\boldsymbol{A L T E R N A T I V A S}$ & $\boldsymbol{F R}$ & $\boldsymbol{\%}$ & $\boldsymbol{F R A}$ & $\boldsymbol{\%} \boldsymbol{A}$ \\
\hline SIEMPRE & 12 & 10 & 12 & 10 \\
\hline ALGUNAS VECES & 64 & 53 & 76 & 63 \\
\hline NUNCA & 44 & 37 & 120 & 100 \\
\hline TOTAL & 120 & 100 & 120 & 100 \\
\hline
\end{tabular}

Fuente: Elaboración propia (2018).

En la tabla $\mathrm{N}^{\mathrm{0}} 3$, se presenta la distribución frecuencial y porcentual del indicador construcción colectiva en lo que se indica en opinión del 53\% de los investigados, que solo algunas se aprovecha el potencial cognitivo individual de los estudiantes para fortalecer el comportamiento del grupo, y se aplican teorías que dinamice su construcción colectiva y se crean condiciones ambientales en el aula para vincular a los alumnos en un proceso de interacción constructiva.

Seguido de la alternativa nunca con un $37 \%$ y con el menor porcentaje se ubicó la alternativa siempre con un $10 \%$, respectivamente. Se observa que algunas veces se logra que los estudiantes entre sí y los facilitadores se vinculan en un proceso de construcción colectiva que enriquezca las ideas y permita unificar posiciones a partir de los requerimientos particulares de los participantes y del programa.

\section{Conclusiones}

El manejo constructivo de las técnicas de enseñanza aplicadas por los facilitadores asumió el siguiente comportamiento:

La interacción significativa, algunas veces se utiliza para sistematizar la construcción del aprendizaje de los alumnos, así como el establecer un orden lógico cuando se formula un problema para que puedan deslindar los componentes principales en su formulación, el aprovechar el pensamiento lógico de los estudiantes para explicar los diferentes componentes que estructuran el problema por resolver y procuran crear condiciones para que el estudiante construya su aprendizaje. 
Se constató, que algunas veces se hace uso de la transferencia de experiencia para vincular la naturaleza del problema con experiencia directa de aprendizaje; así como considerar la elaboración de sus propios programas atendiendo el nivel de conocimientos matemáticos de los estudiantes.

Asimismo, se pudo evidenciar que solo algunas veces se aprovecha el potencial cognitivo individual de los estudiantes para fortalecer el comportamiento del grupo, y se aplican teorías que dinamizan su construcción colectiva, así como crear condiciones ambientales en el aula para vincular a los estudiantes en un proceso de interacción constructiva.

\section{Referencias Bibliográficas}

Arellano, N (2001). El Superaprendizaje. Información en línea. Recuperado en: http://www.redenlaces.cl

Basabe, C (2006). Investigación educativa e integral Editorial Limusa. Colombia.

Brown, J. (2009). Teorías Cognoscitivas. Editorial Mc. Graw Hill. México.

Cárdenas, N. (2005). El arte de enseñar con todo el cerebro. Caracas. Edit. Biosfera.

Hernández, Fernández y Baptista (2014). Metodología de la Investigación. Tercera Edición. México. Editorial Me Graw Hill.

Martínez, M. (2004) Educación y Ciencias Humanas. Postgrado Universidad Nacional Experimental Simón Rodríguez. Revista educación y ciencias humanas. (Año IV) No. 6.

Pisano, B. (2000. La relajación y otras estrategias del superaprendizaje y su valor trascendental en el aula. México. Editorial Trillas

Pulpillo, M. (2001). Aprendizaje Total. España. Editorial. Kapeluz.

Reyes, L. (1996). Un modelo teórico de la acción tutorial en el postgrado. Tesis de grado. URBE. Maracaibo

UNESCO (2005). Hacia una Nueva Etapa de Desarrollo Educativo. La Habana Cuba 